\title{
TRATAMENTO DE RUÍDOS E CARACTERIZAÇÃO DE FISIONOMIAS DO CERRADO UTILIZANDO SÉRIES TEMPORAIS DO SENSOR MODIS ${ }^{1}$
}

\author{
Antonio Felipe Couto Junior², Osmar Abílio de Carvalho Junior ${ }^{3}$, Eder de Souza Martins ${ }^{4}$, Otacílio \\ Antunes Santana ${ }^{5}$, Vinicius Vasconcelos de Souza ${ }^{6}$ e Jose Imana Encinas ${ }^{7}$
}

\begin{abstract}
RESUMO - O Cerrado é formado por um mosaico de fisionomias campestres, savânicas e florestais que possuem um típico ciclo fenológico. Nesse contexto, os dados do MODIS fornecem medidas diárias que permitem monitorar a sazonal fenologia da vegetação. O objetivo deste trabalho foi caracterizar formações savânicas, formações florestais e áreas de cerrado convertido pela ação antrópica, utilizando séries temporais de NDVI e EVI do sensor MODIS, após a suavização de ruídos. A metodologia adotada pode ser subdividida nos seguintes passos: (a) confecção do cubo temporal com NDVI e EVI, onde o perfil em z corresponde à assinatura temporal, (b) tratamento do ruído e (c) detecção das assinaturas temporais. A Transformação pela Fração Mínima de Ruído (MNF) foi aplicada para suavizar ruídos contidos nas séries temporais. Os resultados indicaram que os valores de NDVI foram maiores que os valores de EVI e existiu relação com as estações do ano. As formações florestais foram as que apresentaram maiores valores de NDVI e EVI, possuindo ainda as menores variações entre as estações. As áreas convertidas apresentaram os menores valores dos dois índices, tendo grande queda em seus valores no início da estação seca, provavelmente por se tratar do período de colheita. O estudo concluiu que as séries temporais NDVI e EVI são úteis na diferenciação dos tipos de vegetação.
\end{abstract}

Palavras-chave: Cerrado, MODIS e Série temporal.

\section{DENOISING AND CARACTERIZATION OF CERRADO PHYSIOGNOMIES USING MODIS TIMES SERIES}

\begin{abstract}
Cerrado is formed by a mosaic of grassland, shrubland and woodland physiognomies with a typical phenological cycle. Thus, MODIS data provide daily measurements which allows to monitor the seasonal phenology of the vegetation. The objective of this work was to characterize savanna formations, forest formations and cerrado areas converted by anthropic actions, by using temporal series of MODIS NDVI and EVI after noise reduction. The adopted methodology should be divided into the following steps: (a) elaboration of the temporal cube with NDVI and EVI, in which the z profile corresponds to temporal signature, (b) noise elimination, (c) detection of temporal signature. The Minimum Noise Fraction Transformation (MNF) method was applied to reduce noise in temporal signature. The results showed that the NDVI values were higher than the EVI; and there was a relationship with the seasons of the year. The forest formations presented the highest values of NDVI and EVI, showing the lowest variations among the seasons. The converted areas of Cerrado presented the lowest values in both indices, and their values decreased in the beginning of the dry season, probably because it was the harvesting season. The study concluded that the NDVI and EVI temporal series are useful in differentiation among vegetation types.
\end{abstract}

Keywords:Cerrado, MODIS and Time series.

\footnotetext{
${ }^{1}$ Recebido em 26.08.2008 e aceito para publicação em 18.04.2011.

${ }^{2}$ Universidade de Brasília Campus Planaltina, UNB/FUP, Brasil. E-mail: <antoniofelipejr@gmail.com>.

${ }^{3}$ Departamento de Geografia Universidade de Brasília, UNB, Brasil. E-mail: <osmarjr@unb.br>.

${ }^{4}$ Empresa Brasileira de Pesquisa Agropecuária, Embrapa, Brasil. E-mail: <eder@cpac.embrapa.br>.

${ }^{5}$ Universidade Federal de Pernambuco, UFPE, Brasil. E-mail: <otaciliosantana@gmail.com>.

${ }^{6}$ Programa de Pós-Graduação em Geografia pela Universidade de Brasília, UNB, Brasil. E-mail: <vinicius.vascoza@gmail.com>.

${ }^{7}$ Departamento de Engenharia Florestal, Universidade de Brasília, UNB, Brasil. E-mail: <imana@unb.br>.
} 


\section{INTRODUÇÃO}

O Cerrado ocupa aproximadamente dois milhões de quilômetros quadrados, equivalendo a mais de um quinto da área do país. Estudos realizados por Ribeiro e Walter (1998) mostram que apenas, aproximadamente, $20 \%$ de sua área vegetal está preservada. O Cerrado é considerado a savana mais biodiversa do mundo, com pronunciada heterogeneidade de sua paisagem natural (RATTER et al., 1997; SILVA; BATES, 2002; SILVA et al., 2006). No entanto, possui poucas áreas legalmente protegidas: em torno de 2,2\% de sua extensão (KLINK; MACHADO, 2005). Suas fisionomias variam de formações campestres até formações florestais (RIBEIRO; WALTER, 1998). As espécies lenhosas apresentam comportamentos fenológicos distintos, variando desde espécies sempre verdes a decíduas, e as sempre verdes parecem ter sua produção de folhas limitadas à restrição hídrica sazonal (OLIVEIRA, 2005).

A técnica de sensoriamento remoto mostra-se eficiente no monitoramento da cobertura vegetal, evidenciando as variações temporais, detecção de mudanças, dinâmica sazonal e fenologia (YU et al., 2003). Nesse propósito, o sensor Moderate Resolution Imaging Spectroradiometer (MODIS) é um espectrorradiômetro com 36 bandas, abrangendo as radiações do visível e do infravermelho, permitindo a obtenção de dados relacionados à vegetação, cobertura terrestre, nuvens e aerossóis, incidência de fogo, neve e cobertura de gelo no mar. Esse sensor está a bordo das plataformas TERRA e AQUA, lançadas, respectivamente, em 1999 e 2002. As imagens desse sensor permitem o monitoramento da cobertura global quase em tempo real, possuindo as seguintes resoluções: espacial (250, 500 e $1.000 \mathrm{~m}$ ), espectral (545 a $14.385 \mathrm{~nm}$ ) e temporal (de 1 a 2 dias) (JUSTICE; TOWNSHEND, 2002).

Entre os produtos MODIS disponíveis, encontram-se os índices de vegetação Normalized Difference Vegetation Index (NDVI) e Enhanced Vegetation Index (EVI) gerados automaticamente e disponibilizados na forma de mosaicos. Esses índices estão correlacionados com parâmetros biofísicos da vegetação, possibilitando a estimativa de parâmetros como: índice de área foliar, biomassa vegetal, produtividade e atividade fotossintética (JUSTICE; TOWNSHED, 2002). Em relação à caracterização sazonal da vegetação, Liesenberg et al. (2007) concluíram que não existem diferenças significativas entre as plataformas TERRA e AQUA.
O objetivo deste trabalho foi caracterizar formações savânicas, formações florestais e áreas de cerrado convertido pela ação antrópica, utilizando séries temporais de NDVI e EVI do sensor MODIS, após a suavização de ruídos normalmente presentes nesse tipo de imagem.

\section{MATERIAL E MÉTODOS}

\section{1. Área de estudo}

A área de estudo está dentro de região do EcoMuseu do Cerrado, que possui uma superfície de $8.100 \mathrm{~km}^{2}$, localizada entre os paralelos $15^{\circ} 21^{\prime}$ e $16^{\circ} 21^{\prime}$ de latitude Sul e os meridianos $48^{\circ} 04^{\prime}$ e $49^{\circ} 14^{\prime}$ 'de longitude Oeste, inserido na zona central do Bioma Cerrado (Figura 1). O EcoMuseu pertence ao Estado de Goiás, sendo composto por sete municípios: Abadiânia, Águas Lindas do Goiás, Alexânia, Cocalzinho, Corumbá de Goiás, Pirenópolis e Santo Antônio do Descoberto. Apresenta 55\% da sua área como prioritárias para a conservação da biodiversidade (BRASIL, 1999; NOBREGA; IMAÑA, 2006). No entanto, apenas 0,3\% da área está inserida como proteção integral, pertencente ao Parque Estadual dos Pirineus com aproximadamente 2.800 ha (IMAÑA et al., 2004).

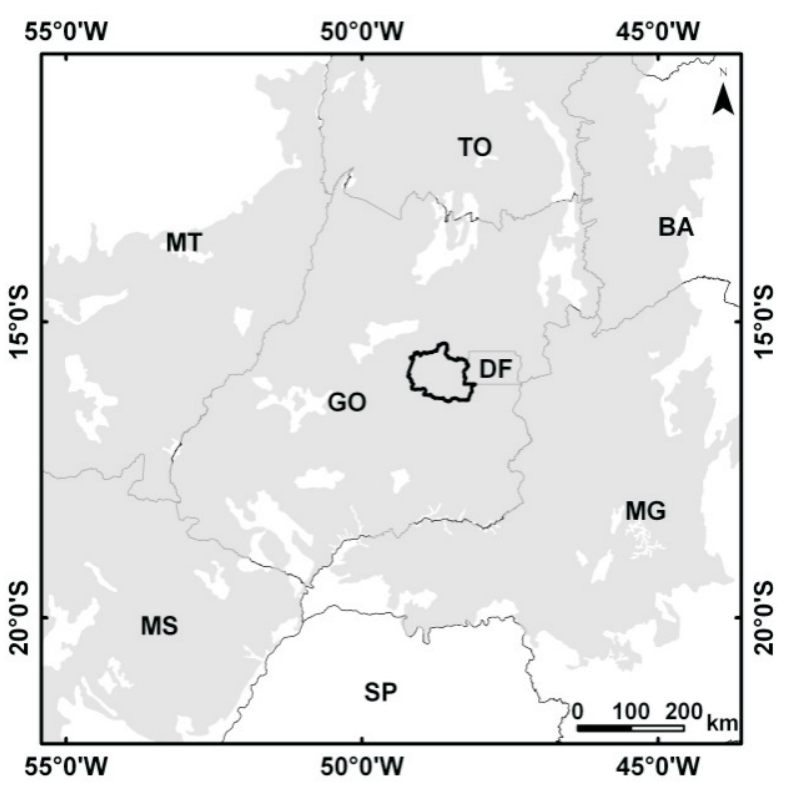

Figura 1 - Localização da região do EcoMuseu do Cerrado (GO), adjacente ao DF, inserido no Bioma Cerrado (área hachurada).

Figure 1 - Location of Cerrado EcoMuseum, Goias state, close to the Federal District, inside of the Cerrado Biome (hatching area).

Revista Árvore, Viçosa-MG, v.35, n.3, Edição Especial, p.699-705, 2011 


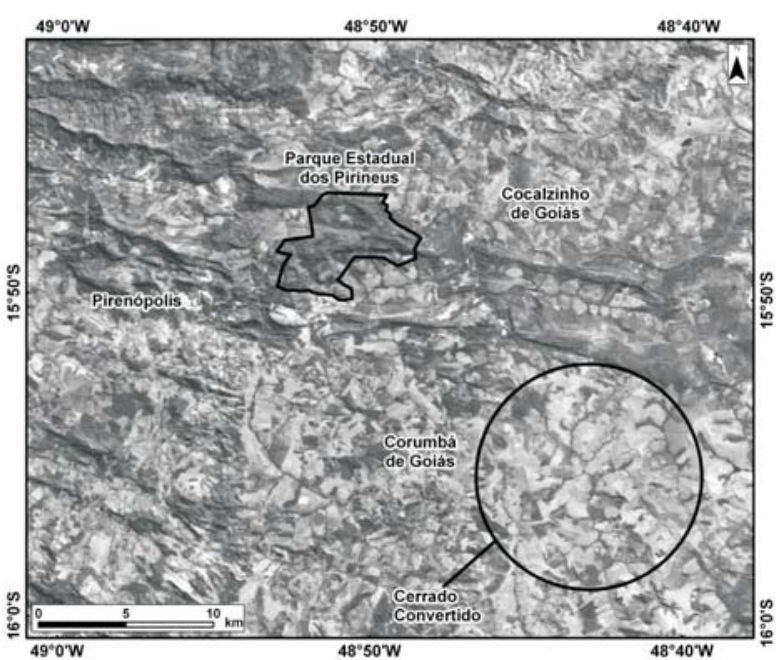

Figura 2 - Visualização do Parque Estadual dos Pirineus (PEP), localizado na parte central e de áreas de cerrado convertido pela ação antrópica (parte inferior), na Imagem CBERS, de 8 de agosto de 2006.

Figure 2 - The Pirineus State Park in the central area and cerrado areas converted by anthropic action (lower right), in CBERS image, August $8^{\text {th }} 2006$.

\subsection{Aquisição dos dados MODIS}

Neste estudo foi utilizada uma série temporal do produto MOD13, que contém os índices de vegetação NDVI e EVI com resolução espacial de 250 m, relativo ao ano de 2005, com uma variação de 16 dias entre cada uma delas, totalizando 23 cenas. Os índices de vegetação minimizam as interferências relativas às variações provenientes do ângulo solar e dos efeitos atmosféricos, observados em dados multitemporais. Enquanto o NDVI é sensível à clorofila, o EVIémais sensível às variações na resposta estrutural do dossel, incluindo o índice de área foliar (LAI), a fisionomia da planta e a arquitetura do dossel (HUETE et al., 2002). ONDVI apresenta a seguinte formulação (ROUSE et al., 1974):

$$
N D V I=\left(r_{I V P}-r_{V}\right) /\left(r_{I V P}+r_{V}\right),
$$

em que "rIVP” é o valor da reflectância no infravermelho próximo (banda 2, 841-876 nm) e "rV” é a reflectância na faixa do vermelho (banda1, 620-670 nm). Os valores obtidos com o NDVI são contidos em uma mesma escala de valores, entre -1 e 1. A principal crítica em relação ao NDVI é a fraca sensibilidade a valores de índice de área foliar entre 2 e 3 (CURRAN, 1983; PRICE, 1992; LIU; HUETE, 1995). No entanto, esse índice mostra-se adequado para ambientes de cerrado (cerrado típico), cujo valor de IAF fica em torno de 1 (MIURA et al., 2003).
O EVI foi desenvolvido para evidenciar as regiões com alta biomassa, reduzindo a influencia do solo e da atmosfera, sendo expresso pela seguinte formulação (JUSTICE et al., 1998):

$E V I=G \times\left[\left(r_{I V P}-r_{V}\right) /\left(r_{I V P}+C_{1} r_{V}-C_{2} r_{A}+L\right)\right]$

em que:

$\boldsymbol{r}_{\text {IVP }}$ e $\boldsymbol{r}_{\mathrm{V}}$ são as reflectâncias das bandas 1 e 2 do MODIS e o $\boldsymbol{r}_{\mathrm{A}}$, a reflectância da banda 3 (459-479 nm); $\mathbf{C}_{1}$ e $\mathbf{C}_{2}$ coeficientes de resistência atmosférica; $\boldsymbol{L}$ é o fator de correção de brilho do dossel; e $\boldsymbol{G}$ é o fator de ganho. Para o MODIS, esses valores são $\mathbf{C}_{1}=6 ; \mathbf{C}_{2}=7,5$; $\boldsymbol{L}=1$; e $\boldsymbol{G}=2,5$ (JUSTICE et al., 1998; HUETE et al., 1994; 1997).

\subsection{Confecção do cubo temporal dos índices de vegetação}

Para obter as séries temporais dos índices de vegetação, as imagens relativas às diferentes datas foram agrupadas em um cubo temporal. Dessa forma, o cubo apresenta nos eixos " $x$ " $e$ " $y$ ” as coordenadas geográficas e no eixo “z”, a assinatura temporal de NDVI ou EVI (Figura 3). As imagens de NDVI e EVI foram ordenadas no cubo utilizando a sequência dos dias do ano em ordem crescente.

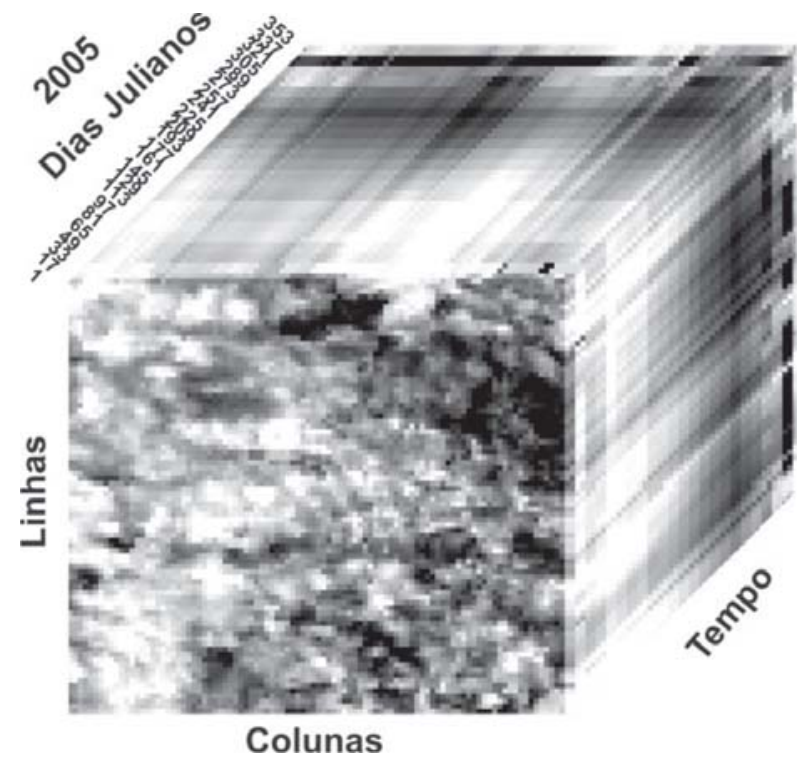

Figura 3 - Imagem do cubo relativo à série temporal NDVI e EVI do sensor MODIS.

Figure 3 - Cube representing NDVI and EVI time serie of the MODIS sensor. 


\subsection{Tratamento do ruído e seleção das assinaturas temporais}

Nos dados da série temporal é comum a presença de ruídos ocasionados por falhas instrumentais, presença de nuvens e sombras, entre outros. Para minimizar a influência desses ruídos foi utilizada a transformação pela fração mínima de ruído (MNF) (GREEN et al., 1988). Esse método foi desenvolvido inicialmente para o tratamento de imagens hiperespectrais; no entanto, mostrou-se adequado para eliminar ou minimizar a interferência de ruídos de outros conjuntos de dados com riqueza de informações como imagens gamaespectrométrica (DICKSON; TAYLOR, 1988, 2000). No processamento de imagens de séries temporais, este método foi aplicado com sucesso na análise temporal de imagens MODIS sobre região de Mata Seca no ambiente de Cerrado (CARVALHO JÚNIOR et al., 2006) e no Parque Nacional da Chapada dos Veadeiros (CARVALHO JÚNIOR et al., 2008).

A transformação MNF é um procedimento estatístico no âmbito da Análise de Principais Componentes (APC) que concilia tanto os procedimentos de segregação da componente ruído quanto também de redução da dimensionalidade dos dados (GREEN et al., 1988). Uma das grandes limitações da APC para a remoção do ruído é que nem sempre se observa nas componentes principais aumento da razão sinal/ruído de forma crescente em relação aos autovalores (GREEN et al., 1988; LEE et al., 1990; CARVALHO JÚNIOR et al., 2003). Dessa forma, a vulnerabilidade da APC para a eliminação do ruído é resultante da priorização da variância no ordenamento dos dados, em vez da razão sinal/ruído, como é adotado pela transformação MNF.

O procedimento para eliminar os ruídos pelo método MNF possui as seguintes etapas: (a) efetuar a transformação MNF na imagem, (b) identificar pela qualidade da imagem e pelo gráfico de autovalores as bandas relativas ao sinal e ao ruído e (c) efetuar a transformação inversa do MNF utilizando somente as bandas relativas ao sinal. Após a transformação inversa do MNF foram selecionadas assinaturas temporais para as formações savânicas e florestais, dentro do PEP, e de cerrado convertido pela ação antrópica em áreas do seu entorno.

\section{RESULTADOS E DISCUSSÃO}

O emprego da técnica da transformação inversa MNF permitiu uma suavização dos ruídos dos perfis temporais, tanto do NDVI quanto do EVI (Figura 4). Para as formações florestais (Figura 4), o tratamento foi capaz de suavizar mais o período compreendido até o dia 100, já nas formações savânicas (Figura 4) as maiores alterações podem ser vistas a partir do dia 250 .

As assinaturas temporais do NDVI e do EVI tratadas pela transformação MNF permitiram uma distinção do comportamento fenológico marcado por intensa atividade fotossintética na época chuvosa e de decréscimo durante o período seco (RATANA et al., 2005). O comportamento sazonal entre as formações florestais, savânicas e áreas convertidas apresentam particularidades que os distinguem. Os maiores contrastes entre as fisionomias estão presentes durante o período da seca, devido à proporção diferenciada de vegetação fotossinteticamente não ativa que ocasiona o decréscimo dos valores dos índices de vegetação.

Tanto os perfis do NDVI quanto do EVI das formações florestais apresentaram valores superiores aos das formações savânicas e áreas alteradas (Figura 5). As formações florestais parecem ser menos influenciadas pela sazonalidade climática, e o NDVI e EVI apresentam-se de maneira mais uniforme ao longo do tempo, corroborando observações realizadas por Ferreira e Huete (2004). Esses autores informaram que esse comportamento pode ser explicado pelo dossel bem desenvolvido das formações florestais.

As áreas convertidas foram as que apresentaram o maior contraste entre as estações nos dois perfis temporais: aproximadamente 0,40 , tanto para o NDVI quanto para o EVI, sendo este o valor também evidenciado por Ratana et al. (2005). A diferença entre os períodos, com rápida queda dos valores, tanto de NDVI quanto de EVI, está relacionada com o pico do plantio, durante a estação chuvosa, e a colheita (FERREIRA; HUETE, 2004) (Figura 5).

Ainda na Figura 5 é possível observar que os valores do NDVI de formações savânicas e cerrado convertido apresentam-se similares até o mês de abril, quando inicia uma queda pronunciada nas áreas de cerrado convertido. Isso pode ser explicado pela atividade fotossintética, que diminui nas áreas convertidas devido às colheitas. Durante todo o ano, os valores de NDVI das formações florestais mantêm-se superiores aos das demais áreas estudadas. Os valores de EVI das áreas convertidas mostramse maiores que os observados nas formações savânicas, podendo superar os valores das formações florestais

Revista Árvore, Viçosa-MG, v.35, n.3, Edição Especial, p.699-705, 2011 


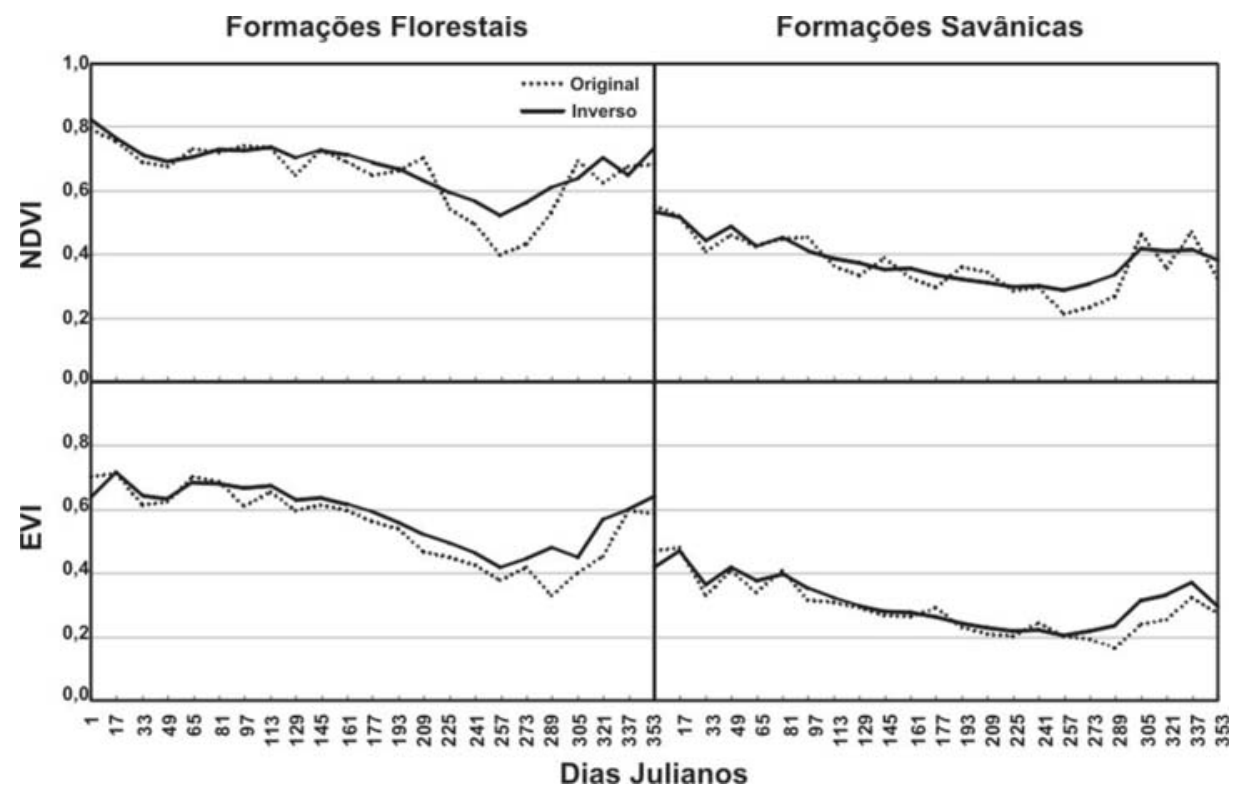

Figura 4 - Série temporal de NDVI e EVI, correspondente a formações florestais e savânicas, antes do tratamento (linha pontilhada) e após a transformação MNF (linha inteira).

Figure 4-NDVI and EVI time series, representing forest and savanna formation, before processing (dotted line) and after MNF transformation (solid line).

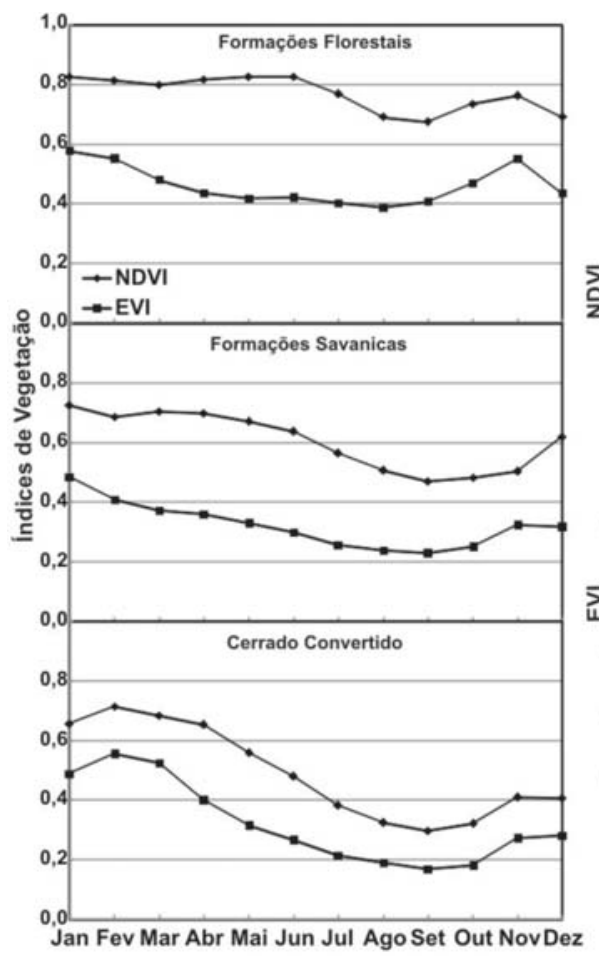

A

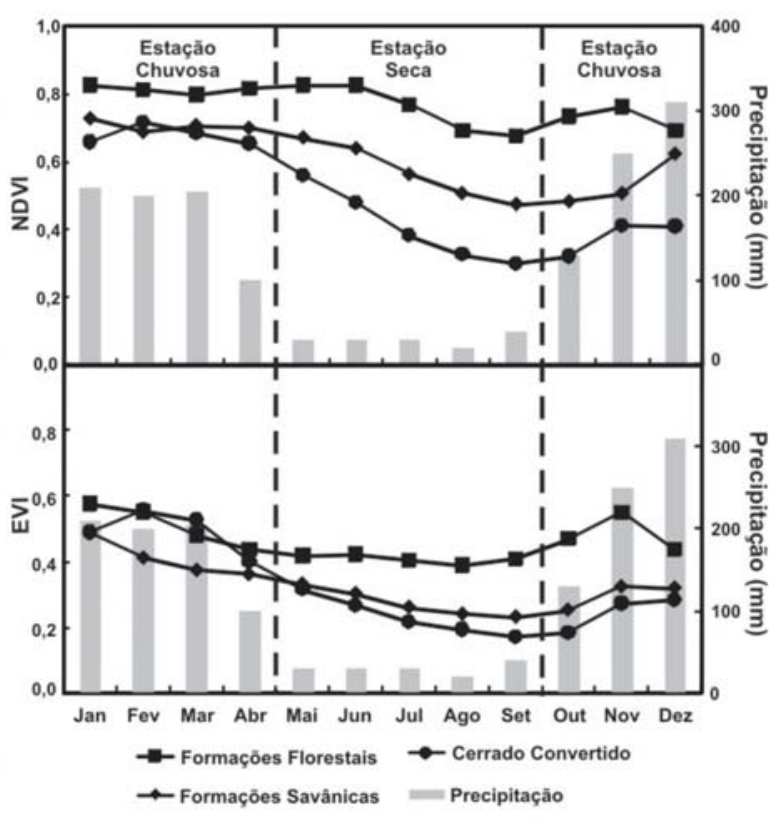

B

Figura 5 - (A) Diferenças entre NDVI e EVI das séries temporais; e (B) Relação entre as séries temporais e precipitação. Figure 5 - (A) Difference between NDVI and EVI of temporal series; (B) Relationship between time series and precipitation. 
durante o período chuvoso. Esse fato mostra-se relacionado ao auge do vigor dos plantios, formando um dossel contínuo e praticamente sem influencia do solo. Por outro lado, durante o período seco, os valores de EVI das áreas convertidas apresentam grande diminuição, mostrando-se inferiores aos valores das formações savânicas, devido à maior exposição do solo após a colheita.

\section{CONCLUSÃO}

Os produtos do MODIS referentes aos índices de vegetação EVI e NDVI são, atualmente, importantes ferramentas de análise e monitoramento da superfície terrestre, em virtude de sua alta resolução temporal e disponibilidade de imagens. Para o Bioma Cerrado, que apresenta intensa fragmentação devido à expansão da agricultura e pecuária, o emprego do sensor MODIS mostra-se adequado ao seu monitoramento. Entretanto, uma das principais limitações para o seu emprego é a baixa relação sinal/ruído. Neste trabalho foi utilizada a transformação MNF para minimizar ou eliminar a presença desses ruídos. Esse método ocasiona a suavização dos espectros, favorecendo a distinção e caracterização das assinaturas temporais das coberturas vegetais.

Entre as fisionomias estudadas, as formações florestais apresentaram os maiores valores de NDVI e EVI e as menores variações, ou seja, mantendo sua cobertura vegetal mais constante ao longo do ano. No período seco, observam-se diferenças marcantes entre as fisionomias devido ao comportamento distinto da atividade fotossintética entre o extrato herbáceo e o arbóreo. As fases do plantio são bem demarcadas nas assinaturas espectrais, permitindo uma caracterização das áreas de uso antrópico com conversão do Cerrado.

\section{AGRADECIMENTOS}

Ao apoio recebido pela Coordenação de Aperfeiçoamento de Pessoal de Nível Superior (CAPES), pelas bolsas de mestrado e doutorado concedidas ao primeiro autor; ao Conselho Nacional de Desenvolvimento Científico e Tecnológico (CNPq), pelo financiamento de bolsas de pesquisa aos professores; e aos revisores da Revista Árvore, pelas correções e sugestões para o aprimoramento deste trabalho.

\section{REFERÊNCIAS}

ALHO, C. J. R.; MARTINS, E. S. De grão em grão, o cerrado perde espaço. Brasília: WWF, 1995. 66p.
BRASIL. Ministério de Meio Ambiente, Recursos Naturais e Amazônia Legal. Ações prioritárias para a conservação da biodiversidade do Cerrado e Pantanal. Brasília: 1999. 26p.

CARVALHO JÚNIOR, O. A. et al. Classificação e eliminação dos ruídos em imagens hiperespectrais pela análise seqüencial da transformação por fração de ruído mínima. Revista Brasileira de Geofísica, v.20, n.1, p.31-41, 2003.

CARVALHO JÚNIOR, O. A. et al. Identificação regional da floresta estacional decidual na bacia do Rio Paranã a partir da análise multitemporal de imagens MODIS. Revista Brasileira de Geofísica, v.24, n.3, p.319-332, 2006.

CARVALHO JÚNIOR, O. A. et al. Classificação de padrões de savana usando assinaturas temporais NDVI do sensor MODIS no parque Nacional Chapada dos Veadeiros. Revista Brasileira de Geofísica, v.26, n.4, p.505-517, 2008.

CURRAN, P. J. Multispectral remote sensing for the estimation of green leaf area index.

Philosophical Transactions of the Royal Society of London, v.309, p.257-270, 1983.

DICKSON, B. \& TAYLOR, G. Noise reduction of aerial gamma-ray survey. Exploration Geophysics, v.29, p.324-329, 1988.

DICKSON, B. \& TAYLOR, G. Maximum noise fraction method reveals detail in gamma-ray surveys. Exploration Geophysics, v.31, n.1, p.73-77, 2000.

FERREIRA, L. G.; HUETE, A. Assessing the seasonal dynamics of the Brazilian Cerrado vegetation through the use of spectral vegetation indices. International Journal of Remote Sensing, v.25, n.10, p.1837-1860, 2004.

GREEN, A. A. et al. Transformation for ordering multiespectral data in terms of images quality with implications for noise removal. IEEE

Transactions on Geoscience and Remote Sensing, v.26, n.1, p.65-74, 1988.

HUETE, A. R. et al. Overview of the radiometric and biophysical performance of the modis vegetation indices. Remote Sensing of Environment, v.83, n.1, p.195-213, 2002. 
HUETE, A. R. et al. A comparison of vegetation indices over a global set of tm images for EOSMODIS. Remote Sensing of

Environment, v.59, n.3, p.440-451, 1997.

HUETE, A.; JUSTICE, C.; LIU, H. Development of vegetation and soil indices for MODIS-EOS. Remote Sensing of Environment, v.49, p.224-234, 1994.

IMAÑA-ENCINAS, J.; NÓBREGA, R. C.; COUTO JUNIOR, A. F. Sugestão de criação de uma área de preservação ambiental na região do EcoMuseu do Cerrado. Boletim do Herbário Ezechias Paulo Heringer, v.14, p.22-32, 2004.

JUSTICE, C. O. et al. The Moderate Resolution Imaging Spectroradiometer (MODIS): land remote sensing for global change research. IEEE Transactions on Geoscience and Remote Sensing, v.36, n.4, p.1228-1247, 1998.

JUSTICE, C. O.; TOWNSHEND, J. R. G. Special issue on the moderate resolution imaging spectroradiometer (MODIS): a new generation of land surface monitoring. Remote Sensing of Environment, v.83, n.1, p.1-2, 2002.

KLINK, C. A.; MACHADO, R. B. Conservation of Brazilian Cerrado. Conservation Biology, v.19, p.707-713, 2005.

LEE, J. B.; WOODYATT, S.; BERMAN, M. Enhancement of high spectral resolution remote sensing data by a noise - Adjusted principal components transform. IEEE Transactions on Geoscience and Remote Sensing, v.28, n.3, p.295-304, 1990.

LIESENBERG, V.; PONZONI, F. J.; GALVAO, L. S. Análise da dinâmica sazonal e separabilidade espectral de algumas fitofisionomias do cerrado com índices de vegetação dos sensores MODIS/TERRA AQUA.

Revista Árvore, v.31, n.2, p.295-305, 2007.

LIU, Q.; HUETE, A. A feedback based modification of the NDVI to minimize canopy background and atmospheric noise. IEEE Transactions on Geoscience and Remote Sensing, v.33, p.457-465, 1995.

MIURA, T. et al. Discrimination and biophysical characterization of cerrado physiognomies with EO-1 hyperspectral hyperion. In: SIMPÓSIO BRASILEIRO DE SENSORIAMENTO REMOTO, 9., 2003, Belo Horizonte. Anais... - Belo Horizonte: 2003. p.1077-1082.
NÓBREGA, R. C.; IMAÑA-ENCINAS, J. Uso atual do solo do projeto EcoMuseu do Cerrado. Revista Árvore, v.30, n.1, p.117-122, 2006.

OLIVEIRA, E. L. Fenologia, demografia e características foliares de espécies lenhosas em um cerrado sentido restrito no Distrito Federal e suas relações com as condições climáticas. 2005. 134f. Tese (Doutorado em Ecologia) Universidade de Brasília, Brasília, 2005.

PRICE, J. C. Estimating vegetation amount from visible and near infrared reflectances. Remote Sensing of Environment, v.41, n.1, p.29-34, 1992.

RATANA, P.; HUETE, A.; FERREIRA, L. G. Analysis of Cerrado physiognomies and conversion in the MODIS seasonal-temporal domain. Earth Interactions, v.9, p.1-21, 2005.

RATTER, J. A.; RIBEIRO, J. F.; BRIDGEWATER, $S$. The Brazilian Cerrado vegetation and threats to its biodiversity. Annals of Botany, v.80, p.223-230, 1997.

RIBEIRO, J. F.; WALTER, B. M. T. Fitofisionomias do bioma Cerrado. In: SANO, S. M.; ALMEIDA, S. P. (Eds.) Cerrado ambiente e flora. Planaltina: Embrapa/CPAC, 1998. p.89-166.

ROUSE, J. W. et al. Monitoring vegetation systems in the Great Plains with ERTS. In: EARTH RESOURCES TECHNOLOGY SATELLITE-1 SYMPOSIUM, 1974, Greenbelt. Proceedings... Greenbelt: 1974. p.301-317. (NASA SP-351)

SILVA, F. J. et al. Spatial heterogeneity, land use and conservation in the Cerrado region of Brazil. Journal of Biogeography, v.33, p.536-548, 2006.

SILVA, J. M. C.; BATES, J. M. Biogeographic patterns and conservation in the South American Cerrado: A Tropical Savanna Hotspot.

Bioscience, v.52, p.225-233, 2002.

YU, F. et al. Response of seasonal vegetation development to climatic variations in eastern central Asia. Remote Sensing of

Environment, v.87, n.1, p.42-54, 2003. 
